\title{
LHC sensitivity to singly charged scalars decaying into electrons and muons
}

\author{
Julien Alcaide $\circledast^{1, *}$ and Nicolás I. Mileo ${ }^{2, \dagger}$ \\ ${ }^{1}$ Departament de Física Tèorica, Universitat de València and IFIC, Universitat de València-CSIC, \\ Dr. Moliner 50, E-46100 Burjassot (Valencia), Spain \\ ${ }^{2}$ IFLP, CONICET_Dpto. de Física, Universidad Nacional de La Plata, C.C. 67, 1900 La Plata, Argentina
}

(Received 30 December 2019; accepted 22 September 2020; published 23 October 2020)

\begin{abstract}
Current LHC searches for nonsupersymmetric singly charged scalars, based on two-Higgs-doublet models, in general, focus the analysis in third-generation fermions in the final state. However, singly charged scalars in alternative extensions of the scalar sector involve Yukawa couplings not proportional to the mass of the fermions. Assuming the scalar decays into electrons and muons, it can manifest cleaner experimental signatures. In this paper, we suggest that a singly charged scalar singlet, with electroweak production, can start to be probed in the near future with dedicated search strategies. Depending on the strength of the Yukawa couplings, two independent scenarios are considered: direct pair production (small couplings) and single production via a virtual neutrino exchange (large couplings). We show that, up to a mass as large as $500 \mathrm{GeV}$, most of the parameter space could be excluded at the $95 \%$ C.L. in a highluminosity phase of the LHC. Our results also apply to other frameworks, provided the singly charged scalar exhibits similar production patterns and dominant decay modes.
\end{abstract}

DOI: 10.1103/PhysRevD.102.075030

\section{INTRODUCTION}

A clear evidence of physics beyond the Standard Model (SM) would be doubtlessly the existence of charged scalars [1], as predicted in many extensions of the SM that incorporate, for instance, new weak multiplets to the scalar sector.

The charged Higgs boson present in the two Higgs doublet model (2HDM) provides a widely studied example of a singly charged scalar arising from an additional weak doublet [2-8]. In fact, most of the searches at colliders are based on the 2HDM and, in general, focus on thirdgeneration fermions in the final state. The charged Higgs boson has been searched at the LEP considering the final states involving the decay channels $\mathrm{H}^{+} \rightarrow c \bar{s}$ and $\mathrm{H}^{+} \rightarrow$ $\tau^{+} \nu_{\tau}$ and assuming that these saturate the decay branching ratio. The combination of all the LEP data excludes a charged Higgs boson with mass below $80 \mathrm{GeV}$ within the context of the 2HDM [9]. On the other hand, the searches for the charged Higgs boson at the LHC target masses above the LEP limit and typically, consider that it is produced in top-quark decays or in association with a

\footnotetext{
julien.alcaide@uv.es

mileo@ fisica.unlp.edu.ar
}

Published by the American Physical Society under the terms of the Creative Commons Attribution 4.0 International license. Further distribution of this work must maintain attribution to the author(s) and the published article's title, journal citation, and DOI. Funded by SCOAP ${ }^{3}$. top quark and/or a bottom quark. These searches cover not only the decay channels analyzed at the LEP [10,11], but also the mode $H^{+} \rightarrow t \bar{b}$, when the charged Higgs boson is heavier than the top quark $[12,13]$.

Charged scalars also appear in the models that incorporate weak triplets such as the Georgi-Machacek model [14], in which the scalar sector of the SM is extended with one complex and one real weak triplets, giving rise to both singly and doubly charged scalars. Other scenarios that include an additional weak triplet are the type-II seesaw models [15-19], where the neutral component of the triplet acquires a vacuum expectation value and generates a Majorana mass for the neutrinos. The mass and couplings of the singly charged scalar appearing in the GeorgiMachacek model have been tested at the LHC through the search for resonant $W Z$ production in the fully leptonic final state [20] and also in the decay channel $\mathrm{H}^{+} \rightarrow W^{+} Z$, with the charged Higgs boson produced via a vector boson fusion [21]. The doubly charged scalar has been, in turn, searched for in the decay channel $\mathrm{H}^{++} \rightarrow \mathrm{W}^{+} \mathrm{W}^{+}$[22]. In Ref. [23], a global fit using all LHC searches of charged Higgs scalars has been performed, with the conclusion that the charged member of the triplet is still allowed for masses above $200 \mathrm{GeV}$. On the other hand, the parameter space of the type-II seesaw mechanism has not been fully explored by any LHC analysis, despite the fact that a singly charged scalar within this scenario could lie at the electroweak scale [24].

Finally, weak-singlet singly charged scalars are particularly interesting since they appear in models that generate 
small radiative neutrino masses. Prominent examples of these models are the Zee model [25] and the Zee-Babu model $[26,27]$ in which the neutrino masses are generated at one and two loops, respectively. In these scenarios, the neutrino masses are predicted to be naturally light even if the new scalars lie at or below the $\mathrm{TeV}$ scale [28]. In addition, the $S U(2)_{L} \times U(1)_{Y}$ gauge quantum numbers of the charged scalar weak singlet are the same as those of the right-handed sleptons in supersymmetric models [29] and the singly charged scalar in $L-R$ symmetric models [30], both cases allowing the new scalar to be at the LHC reach.

So far, there are no dedicated searches for the weaksinglet singly charged scalar at the LHC. With this motivation, in this paper, we develop a LHC search strategy to set bounds on the mass of the charged scalar weak singlet, focused in processes with leptons in the final state, since, in principle, these are more promising than those involving quark decay modes due to the impact of the large QCD backgrounds. Although throughout the text, we will concentrate on the weak-singlet singly charged scalar, we provide results in terms of quantities common to any model in which the charged scalar exhibits similar production and decay patterns.

In general, the leptonic interaction of the charged scalar, called from now on $h^{ \pm}$, would be with pairs of leptons with either chirality. Without adding right-handed neutrinos to the field content, ${ }^{1}$ two different combinations of chiralities arise,

$$
\begin{gathered}
\mathcal{O}_{L L}=\bar{\nu}_{L a} e_{L b}^{c} h^{-}+\text {H.c. }+\cdots \\
\mathcal{O}_{L R}=\bar{\nu}_{L a} e_{R b} h^{+}+\text {H.c. }+\cdots,
\end{gathered}
$$

where the subindex $L(R)$ stands for the left-handed (right-handed) component of the field, and the superindex $c$ transforms the given field into its charge conjugate counterpart. In the context of renormalizable theories, the LL operator comes from gauge invariant leptonic interactions of either a weak singlet or a triplet, while the LR operator is realized when the interaction is with a doublet. Furthermore, both operators can also be generated within effective field theories in which the heavy states have been integrated out.

In the specific case of the singlet, the renormalizable Lagrangian describing its Yukawa interactions with leptons is given by

\footnotetext{
${ }^{1}$ If we considered right-handed neutrinos, there would be two additional operators, namely,

$$
\mathcal{O}_{R R}=\bar{\nu}_{R a} e_{R b}^{c} h^{-}+\text {H.c. }+\cdots
$$$$
\mathcal{O}_{R L}=\bar{\nu}_{R a} e_{L b} h^{+}+\text {H.c. }+\cdots,
$$

whose implementation would be equivalent to that of operators LL and LR, respectively.
}

$$
\mathcal{L}_{h^{ \pm}}^{L L}=f_{a b} \bar{\ell}_{L a} \ell_{L b}^{c} h^{+}+\text {H.c. }
$$

where $\ell_{L}$ denotes the lepton doublet and $\ell_{L}^{c}=i \sigma_{2} \ell_{L}^{*}$ its charge conjugate field. The Yukawa coupling $f_{a b}$ (with $a, b=e, \mu, \tau)$ needs to be antisymmetric due to the presence of the Clebsh factor $i \sigma_{2}$. This interaction belongs to the LL category.

Alternatively, the LR interaction of the singlet to leptons can be realized at dimension 5 following the effective Lagrangian:

$$
\mathcal{L}_{h^{ \pm}}^{L R}=\frac{c_{a b}}{\Lambda}\left(\bar{\ell}_{L a} \tilde{H} e_{R b} h^{+}\right)+\text {H.c. },
$$

where $\tilde{H}=i \sigma_{2} H^{*}, c_{a b}$ encodes the information on new degrees of freedom, and $\Lambda$ is the scale of new physics. After spontaneous symmetry breaking, we retrieve the operator LR in Eq. (2) and identify the coupling constant as $g_{a b} \equiv c_{a b} \frac{v}{\Lambda}$, where $v \sim 174 \mathrm{GeV}$ is the Higgs vacuum expectation value fixing the electroweak scale. Even for a $c_{a b}$ of an order 1 , a new physics scale around the $\mathrm{TeV}$ will typically introduce a suppression factor.

We point out that, at leading order, a charged scalar interacting with leptons could be singly produced (via Yukawa couplings) as well as pair produced (via gauge interactions). In particular, a channel with two charged leptons in the final state ( $2 \ell$ channel) will involve both production modes, and their relative contribution depending on the strength of the Yukawa couplings. For sufficiently small values, the contribution of the single production can be neglected, and in fact, this is the approach followed in Ref. [31]. As the Yukawa couplings grow, however, both contributions start to compete which makes it troublesome to obtain conclusions from a search strategy based on this final state. Therefore, we also analyze the three charged lepton channel ( $3 \ell$ channel), which is more suitable to explore the large Yukawa coupling scenario since it involves only the single production mechanism, via the virtual neutrino exchange.

Even when there are no dedicated searches of the singly charged weak singlet at the LHC, the LEP and LHC searches for charged Higgs bosons and sleptons could, in principle, provide bounds. The LEP limits on a charged Higgs boson assume that $h^{+}$decays exclusively to $c \bar{s}$ and $\tau^{+} \nu_{\tau}$ and in the case in which $h^{+}$can decay significantly into electrons and muons, it is shown in Ref. [31] that the combination of these limits with the slepton search results only exclude masses below $65 \mathrm{GeV}$. On the other hand, the current LHC searches for charged Higgs bosons consider production mechanisms and decay modes involving only third generation fermions and then do not impose relevant constraints on a charged scalar decaying mostly into electrons and muons. This conclusion is even more radical if we assume that the charged scalar does not interact with quarks. Regarding the slepton searches at the LHC [32-34], those performed in the dilepton mode $\left(e^{+} e^{-}\right.$and $\left.\mu^{+} \mu^{-}\right)$ 
impose bounds in the plane $m_{\tilde{\chi}^{0}}-m_{\tilde{\ell}}$ for right-handed, lefthanded, and both right- and left-handed selectron and smuon production. From these bounds, only those corresponding to a massless neutralino and right-handed sleptons could be applied to our case. In Ref. [32], for example, masses above $\sim 380 \mathrm{GeV}$ are excluded. However, this bound is obtained by assuming degenerate selectrons and smuons, while in our case, the dilepton mode originates from a single charged scalar, which reduces the cross section of the process considerably and then weakens the limit. We note that these slepton searches do not consider the virtual lepton exchange production mode. In addition, the LHC searches of staus in the ditau mode can also impose constraints on the branching ratio of the singly charged scalar into taus. Even when the current limits are not very restrictive [35], they are very useful in order to cover the region of small decay branching ratio of $h^{ \pm}$into electrons and muons, where the search strategies based on the dilepton channel lose sensitivity. However, in this paper, we will consider only electrons and muons in the final state and leave the analysis of topologies involving tau leptons as future work.

Finally, bounds can also be set from inclusive analyses that look for final states with three charged leptons. Several searches has been performed in this regard, both by the ATLAS and the CMS Collaborations [36-38]. These model independent searches, being theoretical unbiased, provide their limits in terms of a broad variety of kinematic variables and might not be very efficient in looking for particular new physics. Moreover, they make use of data samples corresponding to low integrated luminosity. Nevertheless, in this paper, we have checked the extent of the bounds on the parameter space of the singly charged scalar that can be obtained with these searches. Note that their results would only apply to the virtual lepton exchange production mode and thus would become more important for large Yukawa couplings.

Our paper will be laid out as follows. In Sec. II, we discuss the production patterns of the charged scalar as well as its decay modes. In Sec. III, we describe the strategy performed in the $2 \ell$ channel and set bounds on the mass of the scalar and its branching ratio to electrons and muons. In Sec. IV, we present the analysis in the $3 \ell$ channel and set limits on the Yukawa coupling and the mass of the scalar. Subsequently, we compare our findings using the LR operator with those obtained with the LL operator. Finally, we conclude in Sec. V.

\section{PRODUCTION AND DOMINANT DECAY MODES}

The production of a charged scalar that does not interact with quarks will depend exclusively on its interactions with the gauge bosons and the leptons. The quantum numbers of the charged scalar under the SM gauge group completely determine the former while only set the tree-level structure of the latter but not its strength. In the case of the weak singlet, for example, the gauge symmetry enforces the renormalizable coupling $f_{a b}$ to be antisymmetric [see Eq. (3)]. However, the strength of these couplings remains undetermined and must be constrained experimentally. For instance, since the interaction $\bar{\ell}_{L} \ell_{L}^{c} h^{+}$induces charged lepton flavor violation, the couplings $f_{a b}$ will receive stringent constraints from lepton rare decays, which can be summarized in the requirements $\left|f_{e \mu} f_{\mu \tau}\right| \lesssim \mathcal{O}\left(10^{-2}\right)$, $\left|f_{e \mu} f_{e \tau}\right| \lesssim \mathcal{O}\left(10^{-2}\right)$ and $\left|f_{e \tau} f_{\mu \tau}\right| \lesssim \mathcal{O}\left(10^{-5}\right){ }^{2}$ These bounds could be satisfied by considering that all the couplings $f_{a b}$ are highly suppressed and then that the renormalizable interactions involving them are negligible (in this scenario, $h^{ \pm}$could be a long-lived particle). In fact, this is the approach used in Refs. [31,41], where both the interactions of the charged scalar with leptons and quarks are derived from dimension-5 operators. Another possibility would be to set two of the couplings to zero and left the remaining one unbounded. This scenario may be accomplished by imposing a lepton flavor global symmetry, such as $L_{i}-L_{j}$, with $(i, j)=(e, \mu),(e, \tau)$ or $(\mu, \tau),{ }^{3}$ which leads to $f_{i j} \neq 0$ and $f_{a b}=0$ for $a b \neq i j$. In this manner, the above experimental bounds are satisfied with the dominant production and decay modes still driven by the renormalizable interactions of Eq. (3). This will be the framework adopted in this paper, although the results can be applied in general to other models of singly charged scalars with a similar pattern of production and decay channels.

The charged scalar can be produced in pairs through its interactions with the gauge bosons $Z / \gamma$ but can also be radiated from a lepton external leg in $s$-channel diagrams with $Z, \gamma$ or $W$ bosons. This leads to the single production in association with two neutrinos, one neutrino and one charged lepton or two charged leptons. Unlike the pair production, the single production depends on the coupling of $h^{ \pm}$to leptons. The Feynman diagrams corresponding to the different production modes are shown in Fig. 1. Since we are assuming that the charged scalar does not couple to quarks, the dominant decay modes will be $h^{+} \rightarrow \ell^{+} \nu_{\ell^{\prime}}$, with $\ell, \ell^{\prime}=e, \mu, \tau$. For the weak singlet with the

\footnotetext{
${ }^{2}$ These limits have been carried out using the analytic expressions in Ref. [28] with the most updated experimental results $[39,40]$ and considering a conservative value for the mass of the charged scalar of $100 \mathrm{GeV}$.

${ }^{3}$ Lepton flavor global symmetries can appear in different scenarios of particle physics, such as models of neutrino masses and baryogenesis. For instance, it was shown that $L_{e}[42,43]$, $L_{e}-L_{\mu}-L_{\tau}$ [44-46], and $L_{\mu}-L_{\tau}[47,48]$ can lead to normal, inverted, and quasidegenerate neutrino mass orderings, respectively. These symmetries can also be useful to explain the baryon asymmetry of the Universe through the leptogenesis mechanism [49]. Special attention has been given in the literature to baryogenesis models with a $\mu-\tau$ flavor symmetry (see Refs. [50,51] for the particular case of $L_{\mu}-L_{\tau}$ and Ref. [52] for a general review of $\mu-\tau$ symmetries).
} 


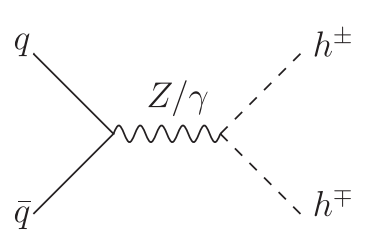

(a)

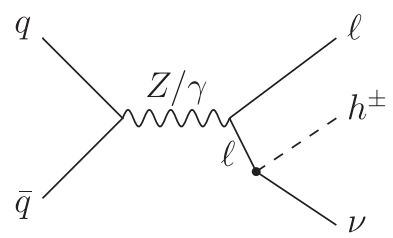

(b)

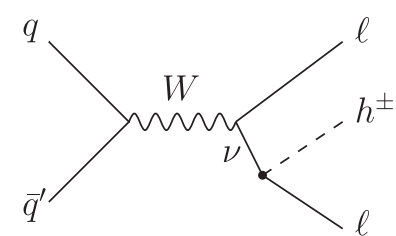

(c)



(d)

FIG. 1. Feynman diagrams involved in the production of the singly charged scalar: (a) corresponds to pair production, (b)-(d) depict different single production modes. The diagrams (a) and (b) contribute to the $2 \ell+E_{T}^{\text {miss }}$ topology, while (c) and (d) lead to $3 \ell+E_{T}^{\text {miss }}$ and $1 \ell+E_{T}^{\text {miss }}$ topologies, respectively.

renormalizable Lagrangian of Eq. (3), we necessarily have $\ell \neq \ell^{\prime}$. Combining the possible decay channels with the production modes displayed in Fig. 1, three different final states arise:

(1) Two opposite-sign leptons plus missing transverse energy, $2 \ell+E_{T}^{\text {miss }}$, associated to diagrams mediated by $Z / \gamma$ [diagrams (a) and (b)].

(2) Three leptons plus missing transverse energy, $3 \ell+E_{T}^{\text {miss }}$, associated to diagram (c).

(3) One lepton plus missing transverse energy, $1 \ell+E_{T}^{\text {miss }}$, associated to diagram (d).

In this study, we focus on the first two, which are, in principle, more promising than the third one that contains just one lepton in the final state. In addition, we consider only electrons and muons in the final state since these flavors can be treated inclusively given their similar cut efficiencies at the LHC. Final states involving tau leptons need to be treated separately, and we left their study as future work. Thus, in the following, the term lepton will describe exclusively electrons and muons.

Since the $3 \ell$ channel originates exclusively from diagram (c) in Fig. 1, the corresponding cross section scales with the coupling of $h^{ \pm}$to leptons as

$$
\sigma_{3 \ell}(f)=f^{2} \times \sigma_{p p \rightarrow h^{ \pm} \ell \ell}(f=1) \times \mathrm{BR}_{e+\mu},
$$

where $\sigma_{p p \rightarrow h^{ \pm} \ell \ell}(f=1)$ is the production cross section for $f=1$, and $\mathrm{BR}_{e+\mu} \equiv \mathrm{BR}_{e}+\mathrm{BR}_{\mu}$ is the decay branching ratio of $h^{ \pm}$into electrons and muons. Clearly, a search strategy based on this channel would lose its sensitivity for decreasing values of the coupling $f$. This is not the case for the $2 \ell$ channel since, besides the production in association with $\ell \nu$, it also involves the pair production, which in fact is the dominant mode for sufficiently small values of the coupling $f$. This can be established more precisely by considering the contribution of the pair production mode to the total cross section in terms of the coupling $f$ for different masses of the charged scalar. From the left panel of Fig. 2, we see that the pair production mode gives the dominant contribution for $f$ below 0.1 . Above this value, the contribution of the single production starts to increase until becoming competitive with the pair production mode for $f \gtrsim 0.6$. The mild dependence with the charged scalar mass is due to the fact that the pair production cross section suffers a higher phase space suppression than the single production.

In summary, for $\mathcal{O}(f) \leq 0.1$ the cross section of the $2 \ell$ channel is fully dominated by the pair production mode and then can be written as

$$
\sigma_{2 \ell}=\sigma_{p p \rightarrow h^{+} h^{-}} \times \mathrm{BR}_{e+\mu}^{2},
$$

while for $\mathcal{O}(f)=1$ the scaling is the one given in Eq. (5) but with $\sigma_{p p \rightarrow h^{ \pm} \ell \ell}$ replaced by $\sigma_{p p \rightarrow h^{ \pm} \ell \nu}$. Taking the above discussion into account, we decided to separate the search strategy according to the strength of the coupling of the charged scalar to leptons. For $f \gtrsim 0.1$, we focus on the $3 \ell$ channel, while for $f<0.1$ we make use of the $2 \ell$ channel. In this manner, we not only retain the sensitivity regardless of the order of magnitude of the coupling $f$ but also are able to translate readily the results obtained for the cross section into results for $\mathrm{BR}_{e+\mu}$ and/or $f$.

Finally, we point out that the signal corresponding to the $2 \ell$ channel can be assumed to emerge from either the LL or LR operators, since they give rise to the same signature.

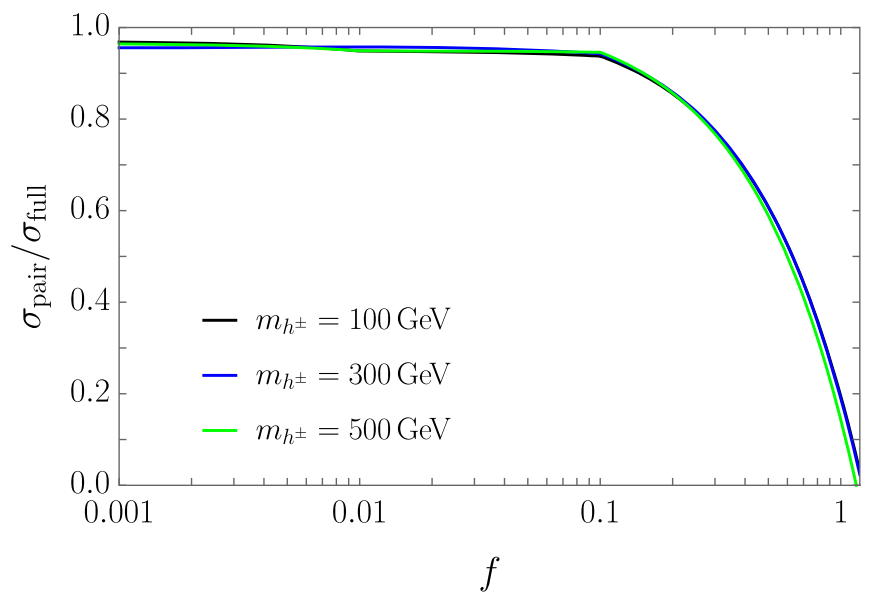

FIG. 2. Contribution of the pair production mode $\left(\sigma_{\text {pair }}\right)$ to the cross section of the $2 \ell$ channel $\left(\sigma_{\text {full }}\right)$. Left panel: behavior of the ratio $\sigma_{\text {pair }} / \sigma_{\text {full }}$ with the coupling $f$ for three different masses of the charged scalar. Right panel: contours of $\sigma_{\text {pair }} / \sigma_{\text {full }}$ in the $f-m_{h^{ \pm}}$plane. 
This is not the case for the $3 \ell$ channel, as we will explain in Sec. IV.

\section{ANALYSIS IN THE TWO LEPTON CHANNEL}

In this section, we focus on the search strategy in the $2 \ell$ channel, following the procedure of Ref. [53]. The topology of the final state consists of two opposite-sign leptons and missing transverse energy. The relevant backgrounds are then Drell-Yan, $t \bar{t}, W W, W Z, Z Z$, and $t W$. We generated all these background processes at leading order for a center of mass energy of $13 \mathrm{TeV}$ with MadGraph_aMC@NLO 2.6 [54] and rescaled their cross sections with different $K$ factors to include the impact of QCD corrections. The parton shower and hadronization were carried out with PYTHIA 8 [55], while the detector response was simulated with DELPHES 3 [56]. In all the cases, we impose the following set of cuts at generator level: $p_{T}^{\ell_{1}}>25 \mathrm{GeV}, p_{T}^{\ell_{2}}>20 \mathrm{GeV},\left|\eta_{\ell}\right|<2.5$, where $\ell_{1}\left(\ell_{2}\right)$ denotes the leading (subleading) lepton. Additionally, for the Drell-Yan process, we set $m_{\ell^{+} \ell^{-}}>100 \mathrm{GeV}$ in order to make the simulation more efficient. The information about the simulation of the different backgrounds is collected in Table I. Regarding the event generation for the signal, we use the package FeynRules [57] to implement the relevant interactions and write them in the UFO format [58]. In order to be conservative, we do not apply any $K$ factor to the signal cross section. The rest of the simulation process proceeds in the same fashion as for the backgrounds. In particular, we demand the signal events to satisfy the same selection cuts at generator level as those imposed on the backgrounds. With the set of cuts imposed at generator level, the pair production cross section at LO of singly charged scalars is $5.5 \mathrm{fb}$, for $m_{h^{ \pm}}=200 \mathrm{GeV}$.

At the reconstruction level, we select events with two opposite-sign leptons, that satisfy the same set of cuts imposed at generator level as well as the requirement $E_{T}^{\text {miss }}>35 \mathrm{GeV}$. The last cut on the total missing transverse energy is useful to reduce the Drell-Yan background which, unlike the signal, does not have large $E_{T}^{\text {miss }}$. Out of this sample, we build the following three observables:

TABLE I. Main backgrounds along with their corresponding cross sections, the applied $K$ factors [59-68], and the number of simulated events used in the analysis of the $2 \ell$ channel.

\begin{tabular}{lccc}
\hline \hline Background & Cross section $(\mathrm{pb})$ & K factor & Simulated events \\
\hline Drell-Yan & 81 & 1.2 & $5.0 \times 10^{7}$ \\
$t \bar{t}$ & 20 & 1.8 & $2.5 \times 10^{7}$ \\
$W W$ & 4.9 & 1.5 & $3.0 \times 10^{6}$ \\
$W Z$ & 2.0 & 1.4 & $1.0 \times 10^{6}$ \\
$Z Z$ & 0.8 & 1.4 & $5.0 \times 10^{5}$ \\
$t W$ & 4.2 & 0.9 & $1.5 \times 10^{6}$ \\
\hline \hline
\end{tabular}

(1) The invariant mass of the opposite-sign lepton pair, $m_{\ell \ell}$.

(2) The transverse mass of the opposite-sign lepton pair, $m_{T \ell \ell}$, defined by

$$
m_{T \ell \ell}^{2}=m_{\ell \ell}^{2}+2\left(E_{T}^{\ell \ell} E_{T}^{\text {miss }}-\vec{p}_{T}^{\ell \ell} \cdot \vec{p}_{T}^{\text {miss }}\right),
$$

where $\vec{p}_{T}^{\ell \ell}=\vec{p}_{T}^{\ell^{+}}+\vec{p}_{T}^{\ell^{-}}$and $E_{T}^{\ell \ell}=\sqrt{\left|\vec{p}_{T}^{\ell \ell}\right|^{2}+m_{\ell \ell}^{2}}$.

(3) The stransverse mass, $m_{T 2}$, defined as

$$
\begin{aligned}
m_{T 2}= & \min _{\vec{q}_{L 1}^{\text {miss }}+\vec{q}_{L 2}^{\text {miss }}=\vec{p}_{T}^{\text {miss }}}\left\{\operatorname { m a x } \left[m_{T}\left(\vec{p}_{T}^{L 1}, \vec{q}_{L 1}^{\text {miss }}\right),\right.\right. \\
& \left.\left.m_{T}\left(\vec{p}_{T}^{L 2}, \vec{q}_{L 2}^{\text {miss }}\right)\right]\right\},
\end{aligned}
$$

with $m_{T}\left(\vec{p}_{T}^{X}, \vec{q}_{X}^{\text {miss }}\right)=\sqrt{m_{X}^{2}+2\left(E_{T}^{X} q_{X}^{\text {miss }}-\vec{p}_{T}^{X} \cdot \vec{q}_{X}^{\text {miss }}\right)}$, where $X$ denotes a visible particle, $E_{T}^{X}$ and $\vec{p}_{T}^{X}$ its transverse energy and momentum, respectively, while $\vec{q}_{X}^{\text {miss }}$ is the part of the missing transverse momentum associated with $X$. The indices $L 1$ and $L 2$ stand for the harder and softer lepton, respectively, and we can safely set $m_{X}=0$ in the definition of $m_{T}$.

Additionally, we consider the observable $S_{T}$ defined as the scalar sum of the transverse momentum of all the leptons in the event. For each observable, $O=m_{\ell \ell}, m_{T \ell \ell}$, and $m_{T 2}$, we build 81 different categories determined by the requirements $O>X$ and $S_{T}>Y$, where $X, Y=$ $100,200, \ldots, 900 \mathrm{GeV}$. Since the $m_{T 2}$ variable is obtained from the transverse masses corresponding to the two leptons arising from the decay of $h^{+}$and $h^{-}$, its distribution exhibits an end point around the charged scalar mass. Therefore, the lowest cut value chosen for this observable in the definition of the categories $(100 \mathrm{GeV})$ is not appropriate for values of $m_{h^{ \pm}}$close to it. For this reason, we added 18 categories corresponding to $m_{T 2}>70 \mathrm{GeV}$ and $m_{T 2}>80 \mathrm{GeV}$ (with $S_{T}>X$ and $X=100, \ldots, 900 \mathrm{GeV}$ ).

We vary the charged scalar mass, $m_{h^{ \pm}}$, between 100 and $500 \mathrm{GeV}$ in steps of $50 \mathrm{GeV}$. For each value of the mass and for each observable, we estimate the lower cross section that can be excluded with a luminosity of $300 \mathrm{fb}^{-1}$ by looking for the category with the largest sensitivity, defined as $S / \sqrt{B}$. Exclusions for intermediate masses are obtained by linear interpolation. The results are shown in Fig. 3. The sensitivity is driven by the $m_{T 2}$ categories in all the considered range of masses, even when it worsens significantly below $m_{h^{ \pm}} \sim 150 \mathrm{GeV}$ and becomes similar to that achieved with the other observables around $m_{h^{ \pm}}=100 \mathrm{GeV}$. With the $m_{T 2}$ categories, it is possible to exclude cross sections ranging from $\sim 30 \mathrm{fb}$ to $0.1 \mathrm{fb}$ for masses between $100 \mathrm{GeV}$ and $500 \mathrm{GeV}$.

It is interesting to interpret the limits given in Fig. 3 in terms of the decay branching ratio of $h^{ \pm}$into electrons and muons. This is easy to accomplish by remembering from Sec. II that the signal cross section factorizes as 




FIG. 3. Bounds on the cross section of pair produced $h^{ \pm}$ decaying into electrons and muons for a total integrated luminosity of $300 \mathrm{fb}^{-1}$. Each curve is obtained by considering only one of the $\mathcal{O}=m_{\ell \ell}, m_{T \ell \ell}, m_{T 2}$ observables as the discriminating variable.

$\sigma\left(p p \rightarrow h^{+} h^{-}\right) \times \mathrm{BR}_{e+\mu}^{2}$. We display the results in Fig. 4 for the most sensitive observable $\left(m_{T 2}\right)$ and two values of the total integrated luminosity, $\mathcal{L}=300 \mathrm{fb}^{-1}$ and $3 \mathrm{ab}^{-1}$. We see from the figure that charged scalars decaying mostly to electrons and muons $\left(\mathrm{BR}_{e+\mu} \sim 0.9\right)$ can be excluded up to $500 \mathrm{GeV}$ with $300 \mathrm{fb}^{-1}$. By increasing the luminosity to $3 \mathrm{ab}^{-1}$, this conclusion can be extended for charged scalars with $\mathrm{BR}_{e+\mu} \geq 0.5$. For a charged scalar decaying only into leptons, the sensitivity gap for $\mathrm{BR}_{e+\mu}<0.3$ could be addressed in principle by considering the ditau channel since $\mathrm{BR}_{\tau}=1-\mathrm{BR}_{e+\mu}$, and then the exclusion limits in terms of $\mathrm{BR}_{\tau}$ translate into an upper limit on $\mathrm{BR}_{e+\mu}$. In fact, by combining our results with the

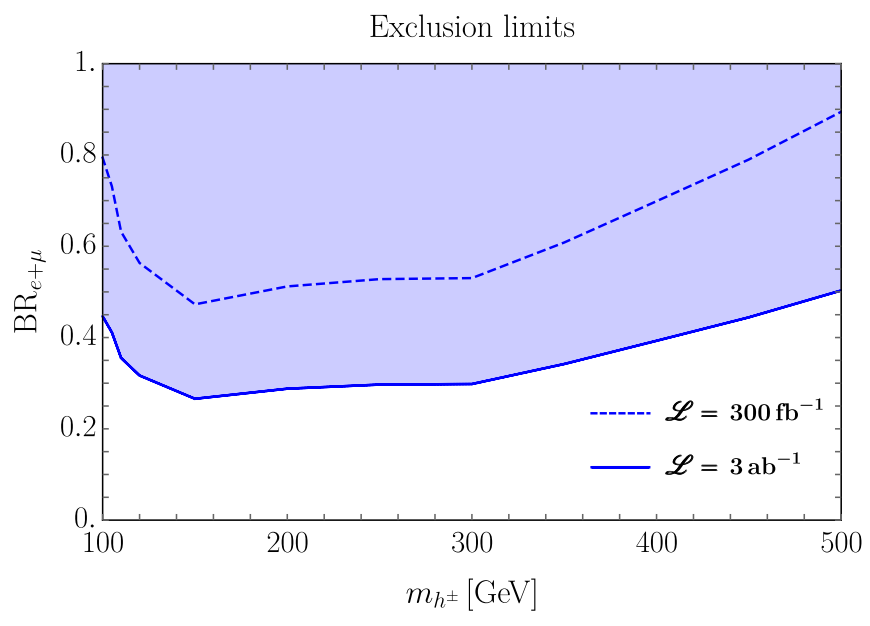

FIG. 4. Bounds in the decay branching ratio of $h^{ \pm}$into electrons and muons with luminosities of $300 \mathrm{fb}^{-1}$ (dashed line) and $3 \mathrm{ab}^{-1}$ (solid line). For this plot, only $\mathcal{O}=m_{T 2}$ was considered, as the sensitivity is driven by this observable. exclusion reported in [31] which is based on the recasting of the analysis of Ref. [69], we can exclude singlet charged scalars with masses below $\sim 280 \mathrm{GeV}$. A search strategy in the ditau channel more focused on the high-mass range could extend this exclusion; however, this is beyond the approach of this paper and is left as future work. Finally, a charged scalar decaying fully into electrons and muons could be ruled out in all the considered mass range $(100 \mathrm{GeV}-500 \mathrm{GeV})$ with a minimum luminosity of $\sim 192 \mathrm{fb}^{-1}$. If a $K$ factor of 1.2 is applied to the signal $[70],{ }^{4}$ this minimum luminosity would reduce down to $\sim 133 \mathrm{fb}^{-1}$.

\section{ANALYSIS IN THE THREE LEPTON CHANNEL}

As previously discussed in Sec. II, the $3 \ell$ channel is more suitable to probe the large Yukawa coupling regime. We again follow the search strategy developed in Ref. [53]. Additionally, we make use of the background presented there, in which a number of events consistent with an integrated luminosity of $3 \mathrm{ab}^{-1}$ was generated at $\sqrt{s}=$ $13 \mathrm{TeV}$. The relevant backgrounds consist of $W Z$, $Z Z, W W W, W W Z, W Z Z, Z Z Z, t t W$, and $t t Z$. At the reconstruction level, we demand electrons (muons) to have $p_{T}>20$ (10) $\mathrm{GeV}$ and $|\eta|<2.5$ (2.6). We construct the following two observables:

(1) $m_{\ell \ell}$, the invariant mass of the two same-sign leptons, and

(2) the transverse mass, defined in the previous section, of the same-sign lepton pair $\left(m_{T \ell \ell}\right)$, as well as the one of the third lepton $\left(m_{T \ell}\right)$.

Like in Sec. III, we additionally consider the auxiliary observable $S_{T}$. Once again, for each observable $O=m_{\ell \ell}$, $m_{T}$ we build 81 different categories with $O>X$ and $S_{T}>Y$, where $X, Y=100,200, \ldots, 900 \mathrm{GeV}$. We remark that, contrary to the pair production channel, in this case, there are two transverse masses, and we demand both of them to simultaneously fulfill the selection cuts. From now on, we will often speak of $m_{T}$ analysis to refer to the combined analysis of the two transverse masses.

Regarding the signal events, we use the package FeynRules [57] to generate the UFO model [58] that implements the relevant interactions. In order to be consistent with the generation of background events in Ref. [53], we choose once again MadGraph 5 [54] and then PYTHIA 6 [71] for parton

\footnotetext{
${ }^{4}$ This $\mathrm{K}$ factor was obtained in Ref. [70] by considering the QCD corrections to pair production of RH sleptons, which have the same quantum numbers than the singly charged scalar.

${ }^{5}$ We do not consider the observable $m_{T 2}$ since, unlike in the $2 \ell$ channel, there is only one source of missing energy in this case. Moreover, in Ref. [53], this observable was constructed taking $L 1$ as the vectorial sum of the two same-sign leptons, while $L 2$ was given by the third one. For this choice, $m_{X}$ in the definition of $m_{T 2}$ [see Eq. (7)] cannot be neglected, as it corresponds to the invariant mass of the two same-sign leptons. This will be enough to make most $m_{\ell \ell}$ and $m_{T 2}$ categories almost identical.
} 



FIG. 5. Exclusion limits at 95\% C.L. of the Yukawa coupling of $h^{ \pm}$decaying into electrons and muons in the $3 l$ channel for a total integrated luminosity of $300 \mathrm{fb}^{-1}$ (left panel) and $3 \mathrm{ab}^{-1}$ (right panel). Colors correspond to: $\mathrm{BR}_{e+\mu}=0.1$ (orange), $\mathrm{BR}_{e+\mu}=0.5$ (green), and $\mathrm{BR}_{e+\mu}=1$ (blue). In black, we show the exclusion limits that can be obtained after recasting the analysis of Ref. [37], based on $20.3 \mathrm{fb}^{-1}$ of data collected at $\sqrt{s}=8 \mathrm{TeV}$, and corresponding to $\mathrm{BR}_{e+\mu}=1$.

showering. We demand the signal events to satisfy the same selection cuts as the background.

We consider the mass of the charged scalar singlet to range between 100 and $500 \mathrm{GeV}$, in steps of $50 \mathrm{GeV}$. Considering solely electrons and muons (plus missing transverse energy) in the final state, the production is such that only the coupling $f_{e \mu}$ is needed. Hereafter, we set $f_{e \mu}=1$ in all the simulations, as the results can be rescaled to any coupling strength following Eq. (5). For each observable and every value of the scalar mass, we look for the category with the largest sensitivity, $S / \sqrt{B}$. By following this procedure, we compute the lowest cross section that can be excluded at the 95\% C.L. Exclusions for intermediate masses are obtained by linear interpolation. For $m_{h^{ \pm}} \gtrsim 250 \mathrm{GeV}$, the sensitivity is slightly driven by $m_{T}$, while for lower values of the mass, the sensitivity of the transverse mass worsens due to the presence of an end point in its distribution around the scalar mass. This makes the observable $m_{\ell \ell}$ the one with the best sensitivity in that region.

The results are shown in Fig. 5 for a total integrated luminosity of $300 \mathrm{fb}^{-1}$ (left panel) and $3 \mathrm{ab}^{-1}$ (right panel). Making use of Eq. (5), we plotted the coupling-mass plane for three different decay branching ratios of the charged scalar, namely $\mathrm{BR}_{e+\mu}=0.1$ (orange), $\mathrm{BR}_{e+\mu}=0.5$ (green), and $\mathrm{BR}_{e+\mu}=1$ (blue).

For comparison, we have also plotted in Fig. 5 the region of the parameter space that can be excluded at the 95\% C.L. by generic searches of final states with three charged leptons (black dashed lines). In order to obtain these limits, we have recast the ATLAS analysis of Ref. [37], based on $20.3 \mathrm{fb}^{-1}$ of data collected at $\sqrt{s}=8 \mathrm{TeV}$. The selection criteria consist of kinematics cuts on the transverse momentum and pseudorapidity of all leptons, as well as isolation cuts. Events are categorized according to several kinematic variables. ${ }^{6}$ The number of predicted background events along with of observed events in several signal regions are provided by the experimental collaboration. Using the $\mathrm{CL}_{\mathrm{s}}$ method [72], we have computed the maximum number of signal events, $s_{\max }$, that is compatible with the observed data given the background. Then, bounds can be obtained by demanding that the number of signal events, calculated with the cross section $\sigma_{3 \ell}(f)$ in Eq. (5), is not larger than $s_{\max }$.

From Fig. 5, we see that if $h^{ \pm}$decays only to electrons and muons, it is possible to exclude the entire range of studied masses for couplings larger than $\sim 1.4$ with a luminosity of $300 \mathrm{fb}^{-1}$. For the high luminosity phase, with $3 \mathrm{ab}^{-1}$, this limit could be extended to couplings as low as $\sim 0.8$. Additionally, smaller branching ratios become more accessible. For instance, a charged scalar with $\mathrm{BR}_{e+\mu}=0.5$ could be probed up to $m_{h} \sim 500 \mathrm{GeV}$ with a coupling $\sim 1.1$. Contrary to the $2 \ell$ channel, in the $3 e$ channel, the analysis based on the LR interaction will report, to some extent, different results than the one considering the LL operator. In particular, the topology with the LR interaction shows a different configuration of the electric charges of the three leptons in the final state (see Fig. 6). For this reason, the observables are constructed with different leptons, and we expect the distributions to disagree from one scenario to the other. In Fig. 7, we show different distributions in both frameworks, for a mass of $200 \mathrm{GeV}$. Notably and in contrast to the case with the LL interaction, $m_{T \ell}$ does not show an end point around the mass of the charged scalar since it is not built with the

\footnotetext{
${ }^{6}$ These observables are: $S_{T}, S_{T}^{\text {hadr }}$ (defined as the scalar sum of the transverse momenta of all jets), the minimum $p_{T}^{\ell}$ of the three leptons, the missing transverse energy, and $m_{\text {eff }}$ (defined as the scalar sum of $S_{T}^{\text {hadr }}, E_{T}^{\text {miss }}$, and the $p_{T}$ of all leptons).
} 


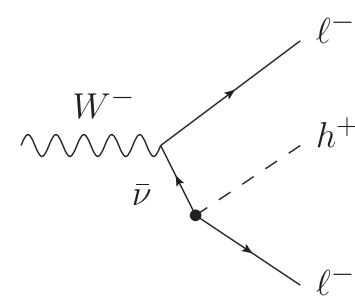

(a) LL interaction

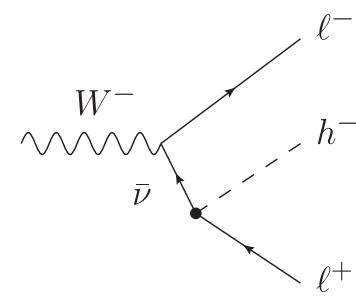

(b) LR interaction
FIG. 6. Feynman diagrams representing the production of the charged scalar through the LL operator (panel a) and the LR operator (panel b).

lepton arising from the decay of the scalar. This feature could help to distinguish one scenario from the other. Additionally, while with the LL operator, two diagrams contribute to the amplitude, there is only one diagram in the LR topology, making its production cross section typically 1 order of magnitude smaller. For instance, for a mass of $200 \mathrm{GeV}$ and coupling $\sim 1$, the production cross section at LO in the context of the LL operator gives $4.7 \mathrm{fb}$, while for the LR operator it reads $0.5 \mathrm{fb}$.

In order to illustrate to what extent the change on the kinematic distributions impacts on the results, let us assume that the Lagrangian in Eq. (2) preserves lepton flavor (that



FIG. 8. Bounds on the cross section of a singly $h^{ \pm}$produced through the LR interaction (dashed blue line) and the LL operator (dashed orange line) for a luminosity of $L=300 \mathrm{fb}^{-1}$, and decaying exclusively to electrons and muons. The theoretical cross section in the LR (solid blue line) and in the LL (solid orange line) case are also shown for reference. The coupling $y$ represents the Yukawa couplings $g(f)$ in the LR (LL) scenario.

is, we only keep the diagonal elements) and redo the whole procedure, fixing the couplings $g_{e e}=g_{\mu \mu}$ for simplicity. Results for the LR operator are depicted in blue in Fig. 8 for a luminosity of $300 \mathrm{fb}^{-1}$, in comparison to those obtained


FIG. 7. Top left panel: Invariant mass distribution of two same-sign leptons in events generated with the LL coupling (orange) and the LR interaction (blue), for $m_{h^{ \pm}}=200 \mathrm{GeV}$. Top right panel: same as before but for the transverse mass distribution of two same-sign leptons. Bottom panel: same as before but for the transverse mass distribution of the single opposite-sign lepton. 
with the LL interaction in orange. The symbol $y$ denotes the Yukawa coupling $g(f)$ in the LR (LL) framework. Solid lines describe the theoretical cross section while dashed lines indicate the maximum cross section that can be probed at the given luminosity. The latter strongly depends on the acceptance of each category and observable. In the whole interval of study, the sensitivity for the LR operator is driven by $m_{\ell \ell}$, whose distribution is very similar to that of the LL interaction. Conversely, the theoretical cross section, as explained above, is typically 1 order of magnitude smaller. For this reason, the exclusion limits will be weaker in this scenario, and for retrieving the same sensitivities achieved with the LL framework, larger couplings or luminosities are needed. In particular, a $h^{ \pm}$ decaying exclusively to electrons and muons through the LR interaction can be excluded with a mass as large as $\sim 170 \mathrm{GeV}$ with a luminosity of $300 \mathrm{fb}^{-1}$, whereas this limit extends to $\sim 370 \mathrm{GeV}$ for the LL interaction.

\section{CONCLUSIONS}

In this paper, we have proposed a search strategy for singly charged scalars focusing in final states with electrons and muons. We have shown that the single production mode through a virtual lepton exchange may have a significant impact on the kinematic distributions in a signature with two leptons for large Yukawa couplings, and then it should be added to the usual pair production mode. Taking this into account, we have split our analysis according to the strength of the interactions of the charged scalar with leptons. For large Yukawa couplings, we have considered a final state consisting of $3 \ell+E_{T}^{\text {miss }}$, which arises from the single production of the charged scalar and its subsequent decay into a lepton and a neutrino. For small Yukawa couplings, the $3 \ell$ channel loses its sensitivity, and then we have considered instead the topology $2 \ell+E_{T}^{\text {miss }}$ since the corresponding cross section is dominated in this scenario by the pair production channel, which is completely determined by the gauge couplings of the charged scalar. In this manner, each topology is driven by only one production mode.
Within the low Yukawa coupling scenario, we have found that a charged scalar decaying exclusively into electrons and muons can be excluded up to masses of $500 \mathrm{GeV}$ with an integrated luminosity of $\sim 200 \mathrm{fb}^{-1}$. In the high luminosity phase, it would be possible to exclude all the studied mass range for any charged scalar with $\mathrm{BR}_{e+\mu} \geq 0.5$. In the large coupling scenario (coupling $\sim 1$ ), the same range of masses can be accessed with a luminosity of $\sim 300 \mathrm{fb}^{-1}$ for couplings $\gtrsim 1.4$, while in the high luminosity phase, this limit could be extended to couplings as low as $\sim 0.8$. Although our results were obtained by assuming that the singly charged scalar is a weak singlet with renormalizable interactions, in principle, they can also be applied to constraint the branching ratio, coupling strength, and mass of singly charged scalars that arise in the context of other models with similar production and decay modes. However, we have shown that special attention is needed when using the results obtained in the $3 \ell$ channel since, in this case, the search strategy is sensitive to the type of interaction of the charged scalar (LL or LR). By recasting our search strategy for the LR interaction, we concluded that the sensitivity decreases in this scenario. In particular, for couplings of order one, a charged scalar decaying fully into electrons and muons via the LL interaction can be excluded up to a mass of $\sim 370 \mathrm{GeV}$, while this limit relaxes to $\sim 170 \mathrm{GeV}$ if the decay proceeds through the LR interaction.

\section{ACKNOWLEDGMENTS}

We thank Mikael Chala and Arcadi Santamaria for useful discussions. N. M. would like to thank Alejandro Szynkman for fruitful discussions regarding the experimental constraints. All Feynman diagrams have been drawn with JaxoDraw [73,74]. This work has been partially supported by CONICET and ANPCyT under Projects No. PICT 2016-0164 and No. PICT 2017-2751 (N. M.) and by the Spanish MINECO under Grant No. FPA201454459-P (J. A.).
[1] J.F. Donoghue and L.F. Li, Phys. Rev. D 19, 945 (1979).

[2] T. D. Lee, Phys. Rev. D 8, 1226 (1973).

[3] T. Lee, Phys. Rep. 9, 143 (1974).

[4] P. Fayet, Nucl. Phys. B78, 14 (1974).

[5] P. Fayet, Nucl. Phys. B90, 104 (1975).

[6] L. Abbott, P. Sikivie, and M. B. Wise, Phys. Rev. D 21, 1393 (1980).

[7] V. D. Barger, J. Hewett, and R. Phillips, Phys. Rev. D 41, 3421 (1990).
[8] G. C. Branco, P. M. Ferreira, L. Lavoura, M. N. Rebelo, M. Sher, and J. P. Silva, Phys. Rep. 516, 1 (2012).

[9] G. Abbiendi et al. (ALEPH, DELPHI, L3, OPAL, and LEP Collaborations), Eur. Phys. J. C 73, 2463 (2013).

[10] G. Aad et al. (ATLAS Collaboration), Eur. Phys. J. C 73, 2465 (2013).

[11] M. Aaboud et al. (ATLAS Collaboration), J. High Energy Phys. 09 (2018) 139.

[12] M. Aaboud et al. (ATLAS Collaboration), J. High Energy Phys. 11 (2018) 085. 
[13] ATLAS Collaboration, Search for charged Higgs bosons in the $H^{ \pm} \rightarrow t b$ decay channel in $p p$ collisions at $\sqrt{s}=$ $13 \mathrm{TeV}$ using the ATLAS detector, CERN Technical Report No. ATLAS-CONF-2016-089, 2016.

[14] H. Georgi and M. Machacek, Nucl. Phys. B262, 463 (1985).

[15] M. Magg and C. Wetterich, Phys. Lett. 94B, 61 (1980).

[16] T. P. Cheng and L.-F. Li, Phys. Rev. D 22, 2860 (1980).

[17] J. Schechter and J. W. F. Valle, Phys. Rev. D 22, 2227 (1980).

[18] G. Lazarides, Q. Shafi, and C. Wetterich, Nucl. Phys. B181, 287 (1981).

[19] R. N. Mohapatra and G. Senjanović, Phys. Rev. D 23, 165 (1981).

[20] M. Aaboud et al. (ATLAS Collaboration), Phys. Lett. B 787, 68 (2018).

[21] G. Aad et al. (ATLAS Collaboration), Phys. Rev. Lett. 114, 231801 (2015).

[22] M. Aaboud et al. (ATLAS Collaboration), Eur. Phys. J. C 79, 58 (2019).

[23] C.-W. Chiang, G. Cottin, and O. Eberhardt, Phys. Rev. D 99, 015001 (2019).

[24] R. Primulando, J. Julio, and P. Uttayarat, J. High Energy Phys. 08 (2019) 024.

[25] A. Zee, Phys. Lett. 93B, 389 (1980); 95B, 461(E) (1980).

[26] A. Zee, Nucl. Phys. B264, 99 (1986).

[27] K. S. Babu, Phys. Lett. B 203, 132 (1988).

[28] J. Herrero-Garcia, M. Nebot, N. Rius, and A. Santamaria, Nucl. Phys. B885, 542 (2014).

[29] S. P. Martin, A supersymmetry primer, in Perspectives on Supersymmetry, Advanced Series on Directions in High Energy Physics Vol. 21 (World Scientific, Singapore, 2010), pp. 1-153; Advanced Series on Directions in High Energy Physics Vol. 18 (World Scientific, Singapore, 1998), pp. 1-98, https://doi.org/10.1142/9789812839657_0001.

[30] P. Fileviez Perez and C. Murgui, Phys. Rev. D 95, 075010 (2017).

[31] Q.-H. Cao, G. Li, K.-P. Xie, and J. Zhang, Phys. Rev. D 97, 115036 (2018).

[32] M. Aaboud et al. (ATLAS Collaboration), Eur. Phys. J. C 78, 995 (2018).

[33] G. Aad et al. (ATLAS Collaboration), Eur. Phys. J. C 80, 123 (2020).

[34] A. M. Sirunyan et al. (CMS Collaboration), J. High Energy Phys. 11 (2018) 079.

[35] ATLAS Collaboration, Search for direct stau production in events with two hadronic tau leptons in $\mathrm{s}=13 \mathrm{TeV} \mathrm{pp}$ collisions with the ATLAS detector, CERN Technical Report No. ATLAS-CONF-2019-018, 2019.

[36] G. Aad et al. (ATLAS Collaboration), Phys. Rev. D 87, 052002 (2013).

[37] G. Aad et al. (ATLAS Collaboration), J. High Energy Phys. 08 (2015) 138.

[38] S. Chatrchyan et al. (CMS Collaboration), J. High Energy Phys. 06 (2012) 169.

[39] A. M. Baldini et al. (MEG Collaboration), Eur. Phys. J. C 76, 434 (2016).

[40] B. Aubert et al. (BABAR Collaboration), Phys. Rev. Lett. 104, 021802 (2010).
[41] E. Boos and I. Volobuev, Phys. Rev. D 97, 095014 (2018).

[42] W. Buchmuller and T. Yanagida, Phys. Lett. B 445, 399 (1999).

[43] F. Vissani, J. High Energy Phys. 11 (1998) 025.

[44] S. Petcov, Phys. Lett. B 110, 245 (1982).

[45] R. Barbieri, L. Hall, D. Tucker-Smith, A. Strumia, and N. Weiner, J. High Energy Phys. 12 (1998) 017.

[46] A. S. Joshipura and S. D. Rindani, Eur. Phys. J. C 14, 85 (2000).

[47] S. Choubey and W. Rodejohann, Eur. Phys. J. C 40, 259 (2005).

[48] W. Rodejohann and M. A. Schmidt, Phys. At. Nucl. 69, 1833 (2006).

[49] M. Fukugita and T. Yanagida, Phys. Lett. B 174, 45 (1986).

[50] T. Ota and W. Rodejohann, Phys. Lett. B 639, 322 (2006).

[51] E. Chun and K. Turzynski, Phys. Rev. D 76, 053008 (2007).

[52] Z.-z. Xing and Z.-h. Zhao, Rep. Prog. Phys. 79, 076201 (2016).

[53] J. Alcaide, M. Chala, and A. Santamaria, Phys. Lett. B 779, 107 (2018).

[54] J. Alwall, R. Frederix, S. Frixione, V. Hirschi, F. Maltoni, O. Mattelaer, H. S. Shao, T. Stelzer, P. Torrielli, and M. Zaro, J. High Energy Phys. 07 (2014) 079.

[55] T. Sjöstrand, S. Ask, J. R. Christiansen, R. Corke, N. Desai, P. Ilten, S. Mrenna, S. Prestel, C. O. Rasmussen, and P.Z. Skands, Comput. Phys. Commun. 191, 159 (2015).

[56] J. de Favereau, C. Delaere, P. Demin, A. Giammanco, V. Lemaître, A. Mertens, and M. Selvaggi (DELPHES 3 Collaboration), J. High Energy Phys. 02 (2014) 057.

[57] A. Alloul, N. D. Christensen, C. Degrande, C. Duhr, and B. Fuks, Comput. Phys. Commun. 185, 2250 (2014).

[58] C. Degrande, C. Duhr, B. Fuks, D. Grellscheid, O. Mattelaer, and T. Reiter, Comput. Phys. Commun. 183, 1201 (2012).

[59] M. Grazzini, S. Kallweit, S. Pozzorini, D. Rathlev, and M. Wiesemann, J. High Energy Phys. 08 (2016) 140.

[60] F. Caola, K. Melnikov, R. Röntsch, and L. Tancredi, Phys. Lett. B 754, 275 (2016).

[61] G. Aad et al. (ATLAS Collaboration), Phys. Rev. Lett. 116, 101801 (2016).

[62] M. Grazzini, S. Kallweit, D. Rathlev, and M. Wiesemann, Phys. Lett. B 761, 179 (2016).

[63] R. Boughezal, J. M. Campbell, R. K. Ellis, C. Focke, W. Giele, X. Liu, F. Petriello, and C. Williams, Eur. Phys. J. C 77, 7 (2017).

[64] V. Ahrens, A. Ferroglia, M. Neubert, B. D. Pecjak, and L. L. Yang, Phys. Lett. B 703, 135 (2011).

[65] M. Czakon, P. Fiedler, and A. Mitov, Phys. Rev. Lett. 110, 252004 (2013).

[66] Q.-H. Cao, arXiv:0801.1539.

[67] S. Frixione, E. Laenen, P. Motylinski, B. R. Webber, and C. D. White, J. High Energy Phys. 07 (2008) 029.

[68] N. Kidonakis, Proc. Sci., DIS2015 (2015) 170.

[69] CMS Collaboration, Search for pair production of tau sleptons in $\sqrt{s}=13 \mathrm{TeV}$ pp collisions in the all-hadronic final state, CERN Report No. CMS-PAS-SUS-17-003, 2017. 
[70] B. Fuks, M. Klasen, D. R. Lamprea, and M. Rothering, J. High Energy Phys. 01 (2014) 168.

[71] T. Sjostrand, S. Mrenna, and P. Z. Skands, J. High Energy Phys. 05 (2006) 026.

[72] A. L. Read, J. Phys. G 28, 2693 (2002).
[73] D. Binosi and L. Theussl, Comput. Phys. Commun. 161, 76 (2004).

[74] D. Binosi, J. Collins, C. Kaufhold, and L. Theussl, Comput. Phys. Commun. 180, 1709 (2009). 